\title{
Review of: "The GET pathway serves to activate Atg32-mediated mitophagy by ER targeting of the Ppg1-Far complex"
}

\author{
Nadine Camougrand ${ }^{1}$ \\ 1 French National Centre for Scientific Research
}

Potential competing interests: The author(s) declared that no potential competing interests exist.

The manuscript that Drs. Onishi and Okamoto proposed focuses on the role of the GET complex in the regulation of mitophagy. The data obtained make it possible to advance the understanding of mitophagy, especially regulation of the phosphorylation/dephosphorylation of the Atg32 protein involving the FAR/Ppg1 complex. The experiments are relatively well conducted, but some aspects can be discussed.

First, there could be homogeneity in the experiments. The protein localization observations by fluorescence are made after $24 \mathrm{~h}$ of growth on a respiratory medium (glycerol); mitophagy is followed by western blots at $24 \mathrm{~h}$ and $72 \mathrm{~h}$, and quantification is done at $72 \mathrm{~h}$. The interaction between Atg32 and Atg11 is measured when the cells are at the start of the exponential phase (OD 1.4). These conditions make it difficult to understand the kinetics of events and the establishment of mitophagy, considering, for example, that the role of the Ppg1 protein is more important before the induction of mitophagy and that the kinase $\mathrm{Ck} 2$ then takes over.

Second, in experiments using and comparing several mutants, the OD does not always provide sufficient information about possible effects of missing proteins (e.g., how mutant cells grow, whether the growth yield and generation time are the same, and whether mutants reach the stationary phase at the same OD as wild type cells). It would be highly useful if the authors could include in their manuscript one control experiment showing and comparing the OD of wild-type and mutant cells at different phases of growth with mitophagy levels simultaneously.

Regarding the experiments showing the interaction between Atg32 and Atg11 with the bioluminescence technique, the ordinate scale (luminescence signal) is low for the WT strain (0.04 max) in Figure 1C. Still, for the WT strain, this measurement varies in Figure 2A (>0.1) and Figure S3A (0.06). For this measurement, the cells were always cultured on glycerol and harvested at the same OD $([1,4]$ i.e., at the start of the exponential phase, conditions for which mitophagy is not induced). It would be important to see 
how this luminescence signal changes during growth because it is supposed to measure the interaction between Atg32 and Atg11 when mitophagy is induced.

In Figure 1A, the phosphorylation of Atg32 appears to be less affected than in the other mutants. The localization of the Far8 protein either to the ER or to the mitochondria is shown by fluorescence experiments, and the quantification graphs are expressed as the percentage of cells with ER or mitochondria localizations. For example, in Figure 3A/B, 80\% of the cells show colocalization of the Sec63mcherry/Far8-3xGFP proteins to the ER, but it seems that in these cells, the Far8 protein is not exclusively localized to the ER (not 100\% colocation); it is difficult to see this on the microscopy images, especially in yeast. Why did you choose the protein Far8 when the two proteins of the FAR complex, which stick to the membranes, are Far9 and Far10? You could have carried out these colocalization experiments with Far9 or Far10. These experiments could be supplemented by a biochemical approach (e.g., OptiPrep gradients), which would show the distribution of this ER/mitochondria protein in the strains tested.

To complete this study, it would be interesting to see what happens in the atg33D mutant, given that the Atg33 protein regulates mitophagy when it is induced at the start of the stationary phase of growth. The authors do not discuss the role that MAMs could play in the transfer of the FAR complex from the mitochondria to the ER or vice versa. It has been shown that proteins of the ERMES complex participate in the regulation of mitophagy.

The last point concerns the quotes used in the manuscript. The purpose of citations is to give the reader the opportunity to consult the pioneering works in the field and take stock of the situation before introducing the original research contained in the manuscript. Unfortunately, recent literature reviews are often cited instead of original studies in this manuscript. In addition, some laboratories practice excessive self-citation.

Examples

On page 2, lines 45-46, mitophagy was described in 2004, 2005, and 2007 by the use of strictly respiratory carbon sources-well before 2018 and 2021, as mentioned in the manuscript.

On page 3, line 55, the work of Innokentev et al. (2020) should be cited.

On page 3, lines 63-72, there are too many citations:

line 64, keep Denic et al., 2012, and add Schuldiner et al., 2005;

line 66, remove Mateja et al., 2015;

lines 68-69: remove McDowell et al., 2020, and Wang et al., 2014;

page 5, line 104, add Aoki et al., 2011

Other comments

On page 11 , lines $243-252$, the observations made are almost identical to those that Innokentev et al. 
(2020) described with Far9 Tom5TA. 